\title{
Força Motivacional, Suporte à Transferência e Impacto do Treinamento no Trabalho
}

Rafaela Moreira ${ }^{1}$, Gardenia da Silva Abbad, Luciana Padovez Cualheta, Elaine Rabelo Neiva

Universidade de Brasília, Brasília, Distrito Federal, Brasil

\section{Palavras-Chave:}

treinamento no trabalho;

motivação;

transferência de treinamento, suporte psicossocial.

\begin{abstract}
Resumo
Há uma preocupação crescente em avaliar efeitos de treinamento no desempenho dos egressos, bem como nos resultados organizacionais. Apesar dos altos investimentos, nem sempre o que é aprendido converte-se em comportamento no trabalho. Estudos demonstram que motivação para aprender e transferir e suporte à aplicação de novas aprendizagens no trabalho são preditores de impacto do treinamento no trabalho. Este estudo objetiva avaliar a influência de percepções de Suporte Psicossocial à Transferência e Força Motivacional sobre Impacto em Amplitude do Treinamento no Trabalho, com base na avaliação de três treinamentos. Participaram desta pesquisa 2.'975 egressos de três treinamentos oferecidos pela Universidade Corporativa de uma empresa pública federal. Foi usada a técnica de modelagem de equações estruturais para testar 5 hipóteses. As escalas utilizadas apresentaram evidências de validade. Os resultados indicaram que Força Motivacional e Suporte Psicossocial à Transferência são preditoras de Impacto do Treinamento no Trabalho e que Força Motivacional é mediadora das relações entre Suporte Psicossocial à Transferência e Impacto do Treinamento no Trabalho. Conclui-se que é preciso evidenciar os benefícios do treinamento para os egressos e organização, estimulando o aumento da motivação para aprender e transferir e que os egressos precisam de suporte de seus superiores e colegas para transferir.
\end{abstract}

Motivation to transfer, Support to transfer and its impacts in transfer of training
Keywords:

work training;

motivation at work;

transfer of training,

psychosocial support.

\begin{abstract}
There is an increasing concern regarding training effects in organizational results. Organizations invest a lot of money in training and often those efforts do not translate in better or improved results. This study aims to identify how support to transfer and motivation to transfer affect transfer of training and the mediation effects among that. Three corporate trainings were evaluated with 2.975 respondents of a public organization in Brazil. Structural equation modeling was used to test five hypotheses, referring to motivation to transfer, support to transfer and transfer of training. Results indicate that instruments are valid and that support to transfer and motivation to transfer predict transfer of training. Also, motivation to transfer mediates the relation among support to transfer and transfer of training. Therefore, it is necessary to evidence the benefits of training, stimulating trainees' motivation to learn and transfer. They should also have support from their superiors and peers to transfer of they have learned.
\end{abstract}

Fuerza motivacional, Soporte de Transferencia y impacto del entrenamiento en el trabajo

\section{Resumén}

Hay una preocupación creciente en evaluar efectos de entrenamiento en el desempeño de los egresados, así como en los resultados organizacionales. Aunque las altas inversiones, no siempre lo que se aprende se convierte en comportamiento en el trabajo. Los estudios demuestran que la motivación para aprender y transferir y apoyar la aplicación de nuevos aprendizajes son predictores de impacto del entrenamiento en el trabajo. Este estudio objetivó evaluar la influencia de percepciones de Apoyo Psicosocial a la Transferencia y de la Fuerza Motivacional en el entrenamiento sobre el Impacto del Entrenamiento en el Trabajo. Participaron de esta investigación 2975 egresados de tres entrenamientos ofrecidos por la Universidad Corporativa de una empresa pública brasileña. Se ha utilizado el análisis de ecuaciones estructurales para probar cinco hipótesis. Las escalas utilizadas presentaron fuertes evidencias de validez. Los resultados indicaron que Fuerza Motivacional y Soporte Psicosocial son predictores de Impacto del Entrenamiento en el Trabajo y que Fuerza Motivacional es mediadora de las relaciones entre Soporte Psicosocial e Impacto del Entrenamiento en el Trabajo. Se concluye que es necesario explicitar los beneficios del entrenamiento para los participantes, estimulando así la motivación para aprender y el soporte de los superiores y colegas a la transferencia.

\footnotetext{
Endereço para correspondência:

Campus Universitário Darcy Ribeiro, Brasília-DF | CEP 70910-900. E-mails: <rafaela.mecm@gmail.com>;

Como citar este artigo:

Moreira, R., Abbad, G.S., Cualheta, L.P., \& Neiva, E.R. (2019). Força Motivacional, Suporte à Transferência e Impacto do Treinamento no Trabalho. Revista Psicologia: Organizações e Trabalho, 19(2), 631-639. doi: 10.17652/rpot/2019.2.15939
} 
Programas de treinamento, desenvolvimento e educação (TD\&E) proporcionam uma série de benefícios para as organizações, especialmente no desenvolvimento de competências profissionais, melhorar no desempenho de tarefas e no incremento de produtividade (Curado, Henriques \& Ribeiro, 2015). Por isso, transferência de treinamento e motivação para transferir tornaram-se temas estratégicos na área de gestão de recursos humanos e vêm ganhando cada vez mais espaço na literatura (Burke \& Hutchins, 2007).

Transferência do treinamento, e o seu construto correlato, impacto do treinamento no trabalho, refere-se a quanto os egressos aplicam eficazmente no ambiente de trabalho os conhecimentos, habilidades e atitudes, adquiridos por meio da ação instrucional (Baldwin \& Ford, 1988; Abbad, Borges-Andrade \& Sallorenzo, 2004). Parte do pressuposto da necessidade do treinando em perceber o quanto os conhecimentos e habilidades adquiridos por meio do treinamento podem ajudá-lo a aperfeiçoar o seu desempenho e serem úteis na resolução de problemas no ambiente de trabalho. Essa crença pode atuar como fator motivacional. Por isso, ao estudar a transferência de treinamento, é importante compreender o quanto o indivíduo está motivado a transferir (Curado et al., 2015).

A motivação para transferir se refere ao esforço despendido pelo indivíduo para aprender os conteúdos de um curso (Santos \& Mognon, 2016) e à direção, intensidade e persistência do esforço para usar, no ambiente de trabalho, os conteúdos aprendidos durante o treinamento (Curado et al., 2015). A motivação para transferir aumenta as chances de a transferência de fato ocorrer (Gegenfurtner, Veermans, Festner, \& Gruber, 2009), é influenciada pelo ambiente e media a relação entre a aprendizagem e a mudança comportamental do indivíduo (Noe, 1986).

Colquitt, LePine e Noe (2000) em uma metanálise sobre motivação para o treinamento identificaram que o suporte dos supervisores, dos pares e um bom clima organizacional estão positivamente relacionados com a motivação para aprender e com a motivação para transferir, garantindo a aplicação dos novos conhecimentos no ambiente de trabalho. Bell, Tannenbaum, Ford, Noe e Kraiger (2017), por sua vez, afirmam que o contexto de trabalho tem relação direta com a eficácia das atividades de treinamento, pois ele afeta a motivação dos funcionários para aprender e transferir os conhecimentos aprendidos.

Bell, et al. (2017) realizaram uma revisão da literatura dos últimos 100 anos na área de T\&D e constataram que suporte dos pares e do supervisor pode determinar até que ponto os egressos irão transferir as competências recém-adquiridas para o contexto de trabalho. Esses autores indicam que quando a cultura organizacional é favorável à transferência, refletindo valores que indicam a importância dos treinamentos para a organização, a motivação para transferir é positivamente afetada. Por outro lado, quando os supervisores são avessos à mudança, os treinandos sentem-se inibidos e pouco motivados para aplicar os novos conhecimentos no trabalho (Gegenfurtner, 2011).

Motivação é um dos tópicos mais estudados em pesquisas internacionais da área. Variáveis motivacionais são preditoras de transferência de treinamento (Al-Eisa, Furayyan, \& Alhemoud, 2009; Chiaburu, Van Dam, \& Hutchins, 2010, Krishnamani \& Haider, 2016), de aprendizagem (Bräten \& Ferguson, 2015; McEown, Noels \& Saumure, 2014) e de intenção para transferir (Hutchins, Nimon, Bates, \& Holton, 2013). Motivação mantêm relacionamentos positivos com suporte de supervisores e pares e exerce o papel de variáveis mediadoras do relacionamento entre suporte, transferência e desempenho do egresso no cargo. Kodwani (2017) assinala que a motivação para transferir é uma variável mediadora (total) do relacionamento entre suporte social e transferência de treinamento.
Reinhold, Gegenfurtner e Lewalter (2018) mostraram que motivação para transferir é mediadora (total) do relacionamento entre suporte e transferência de treinamento e que suporte dos supervisores, pares, sanções e feedbacks/coaching influenciam indiretamente a transferência. Foi também encontrado que suporte influencia diretamente a motivação para transferir aprendizagem para o trabalho. Os autores sugerem mais estudos sobre esse relacionamento, pois motivação varia em magnitude e conteúdo a depender do tipo de suporte (do supervisor ou dos pares) ou da natureza (sanção, restrição versus suporte). Por sua vez, Burke e Hutchins (2007) relatam que as formas de suporte gerencial, tais como discussão sobre as novas aprendizagens, mentorias, envolvimento dos supervisores no treinamento e feedback são reconhecidas pelos funcionários como tendo impacto em sua motivação para transferir.

Diversas variáveis antecedentes apresentam relações positivas com motivação para aprender e para transferir, entre as quais as seguintes: (1) experiências emocionais sobre motivação para aprender (López \& Aguilar, 2013); (2) desenho instrucional sobre motivação para aprender (Wingo et al., 2015); (3) tipo de inscrição do treinando no programa de treinamento (voluntária ou obrigatória) sobre a motivação para transferir (Curado et al. 2015); (4) relacionamento e satisfação instrucional com motivação para transferir (Gegenfurtner, Festner et al., 2009). Além disso, foram encontradas relações negativas entre (5), idade cronológica com autoeficácia (confiança dos trabalhadores sobre as próprias habilidades para aprender) e o valor da aprendizagem (importância atribuída pelos trabalhadores à aprendizagem e às atividades de desenvolvimento, em relação a outros objetivos perseguidos por eles no trabalho), dois componentes do construto de crenças motivacionais sobre aprendizagem (Kochoian, Raemdonck, Frenay, \& Zacher, 2016).

Zerbini e Abbad (2011) apontam, que nas principais pesquisas sobre suporte e clima para transferência, o suporte de colegas, da gerência e da organização como um todo, facilita a aquisição de conhecimentos, habilidades e atitudes em treinamentos, e a posterior transferência desses conteúdos para o ambiente de trabalho. Entretanto, há poucas pesquisas nacionais que relacionam variáveis motivacionais, de suporte e impacto do treinamento no trabalho e não foram encontrados estudos sobre o papel mediador de motivação, tampouco sobre o tipo de treinamento como variável moderadora desses relacionamentos, apesar de estudos recentes, como o realizado por Lacerenza, Reyes, Marlow e Salas (2017), indicarem a importância das variáveis de desenho e implementação de treinamentos na predição da eficácia de treinamentos em diferentes níveis de análise. Esse tipo de estudo possibilita investigar os relacionamentos diretos e indiretos entre variáveis relevantes em uma organização nacional, assim como indicar melhorias no desempenho dos sistemas de TD\&E e do contexto em que se inserem os treinamentos.

Diante desse contexto, o objetivo deste estudo é avaliar a influência de percepções de Suporte Psicossocial à Transferência (SUP) e Força Motivacional (FatMotiva) sobre Impacto do Treinamento no Trabalho (IMTT), a partir da avaliação de três treinamentos corporativos. Além disso, pretende-se avaliar a relação de mediação entre as variáveis. Neste estudo, foi adotado um modelo de investigação para testar as relações de predição direta e mediação por meio de modelagem de equações estruturais. Após a revisão de literatura feita, a Figura 1 mostra o modelo para testar relações de predição de impacto e a Figura 2 mostra o modelo para investigar se suporte é variável mediadora da relação entre Força motivacional e Impacto do treinamento no trabalho. 


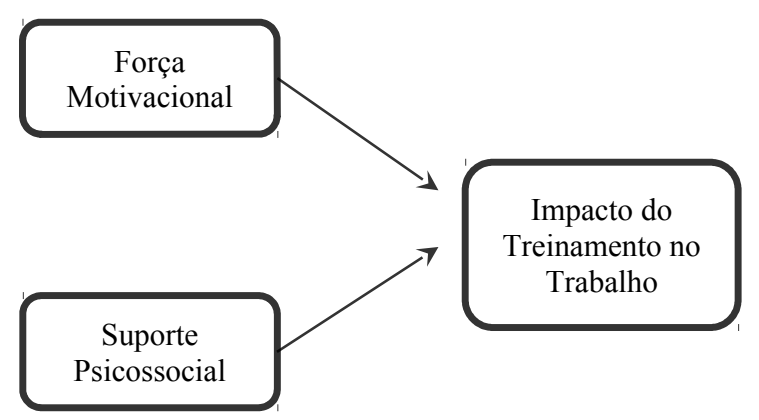

Figura 1. Modelo preditivo proposto

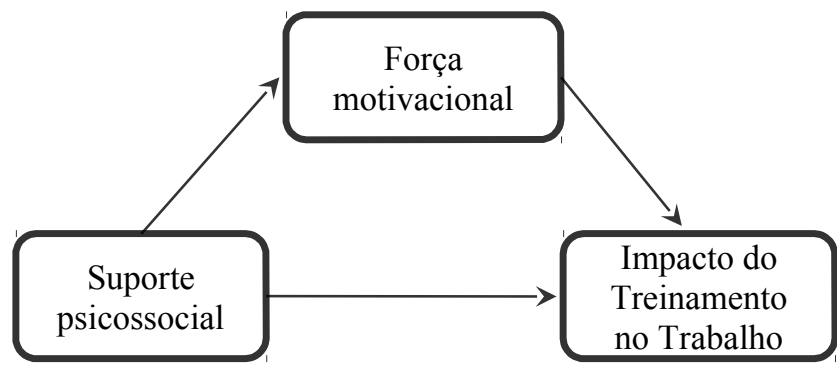

Figura 2. Modelo de Mediação proposto

Com base nos achados científicos discutidos, cinco hipóteses foram testadas na presente pesquisa: H1: Suporte psicossocial à transferência de treinamento afeta positivamente a força motivacional; $\mathrm{H} 2$ : Força motivacional prediz positivamente o impacto de treinamento no trabalho; H3: Suporte psicossocial prediz positivamente o impacto de treinamento no trabalho; H4: Força motivacional media a relação entre suporte psicossocial e impacto de treinamento no trabalho; $\mathrm{H} 5$ : $\mathrm{O}$ tipo de treinamento modera as relações entre suporte psicossocial, força motivacional e impacto do treinamento no trabalho.

\section{Método}

\section{Delineamento}

Estudo descritivo, de abordagem quantitativa, com base em fontes primárias, tendo sido adotado procedimentos descritivos e inferenciais para caracterizar as variáveis e testar as hipóteses do estudo, por meio da técnica estatística multivariada e da modelagem por equações estruturais.

\section{Contexto da Pesquisa}

Este estudo foi realizado na Universidade Corporativa de uma empresa pública federal prestadora de serviço público, com mais de cem mil empregados, com sede em Brasília e estruturas regionais alocadas em quase todos os municípios brasileiros. A escolha da organização foi por conveniência, levando em consideração o critério de disponibilidade e consentimento para a realização da pesquisa, com acesso à pessoas para fornecimento de informações e à documentos organizacionais considerados relevantes ao estudo, dentre os quais: desenho instrucional dos treinamentos, características dos participantes, contextualização dos treinamentos e materiais relacionados ao treinamento em si.

A escolha dos treinamentos avaliados neste estudo foi feita com base em critérios de que previam a coleta de dados em amostras de participantes treinamentos que diferissem quanto aos resultados esperados e público-alvo. Por isso, dois dos treinamentos escolhidos, Agente de Melhorias e Gestão do Absenteísmo e o Incentivo à Presença Produtiva, destinados a gerentes e a líderes, visavam melhorar resultados organizacionais, enquanto o terceiro, destinado a todos os empregados, visava, em curto-prazo, a mudança de comportamento do egresso do treinamento no trabalho, necessária à implantação de um sistema de comunicação e tramitação obrigatória de documentos.

\section{Caracterização dos treinamentos avaliados}

\section{a. Agente de Melhorias}

O treinamento Agente de melhorias, de natureza presencial e com duração de 16 horas, foi realizado em dois dias, com 863 participantes. O objetivo geral deste treinamento é desenvolver competências gerenciais e de liderança para implantar e gerenciar práticas de melhoria contínua em processo de trabalho, visando ao aumento da eficiência das equipes e da organização. Em relação às estratégias instrucionais, observou-se na documentação do curso que predominaram aulas expositivas apoiadas por slides e por um guia contendo instruções e orientações sobre o que tutor deve realizar em sala de aula. Os materiais instrucionais indicam a utilização de exemplos extraídos das situações de trabalho, como recursos didáticos de apoio às discussões sobre os temas abordados no treinamento.

\section{b. Gestão do Absenteísmo e o Incentivo à Presença Produtiva}

Trata-se de um treinamento autoinstrucional, à distância (EaD), de 16 horas, ofertado por diversas regionais da organização a 2.055 funcionários por meio da plataforma Moodle. O objetivo geral do treinamento é desenvolver as competências gerenciais dos líderes da organização para uma melhor gestão das ausências e presenças no trabalho, e a obtenção de efeitos positivos sobre o desempenho dos egressos (impacto do treinamento no trabalho) e sobre os resultados organizacionais (redução do absenteísmo). Os materiais do curso são disponibilizados ao aluno pelo Moodle. Os recursos utilizados são: materiais escritos, exercícios de retenção de conteúdos com questões objetivas e palavras-cruzadas. Sobre as interações presentes no treinamento, a única adotada pelo curso é a interação aluno-material (aluno-máquina), uma vez que não há tutores e os fóruns não são utilizados. Por fim, quanto aos exercícios e avaliações realizados, observou-se que ambos mensuram somente aspectos cognitivos, de conhecimentos básicos, conceituais e de procedimentos. Os exercícios são realizados após o término de cada módulo e a avaliação de aprendizagem é única e realizada ao final do treinamento. Vale ressaltar que a avaliação final permite três tentativas de respostas. Os critérios de aprovação no treinamento são: a leitura do material ofertado e a realização das atividades avaliativas (exercícios e avaliação de aprendizagem).

\section{c. Sistema Eletrônico de Informação (SEI)}

Trata-se de um treinamento autoinstrucional, a distância (EaD), de oito horas, ofertado por diversas regionais da organização a um quantitativo de 16.218 funcionários, por meio da plataforma Moodle. O objetivo geral do treinamento é capacitar todos os empregados da organização para a operação do Sistema Eletrônico de Informação (SEI), que seria, nos meses seguintes à coleta de dados desta pesquisa, de uso obrigatório para a tramitação de todas as comunicações e decisões administrativas da organização. 0 curso engloba um conjunto de módulos e funcionalidades que tem a pretensão de promover, à longo-prazo, um aumento da eficiência 
administrativa. É composto por estratégias instrucionais interativas, adequadas aos seus objetivos, incluindo exercícios programados e exemplos. Os feedbacks, tanto dos exercícios quanto das avaliações, são informativos e oferecidos automática e imediatamente após cada resposta do participante. Por fim, para aprovação no curso é necessário atingir a pontuação de $70 \%$ nas avaliações.

Em suma, os treinamentos Agente de Melhorias e Gestão do Absenteísmo foram desenhados para gerentes e líderes da organização com a finalidade de melhorar resultados organizacionais, enquanto que o treinamento do SEI. foi desenhado para todos os servidores, com a finalidade de capacitá-los em conteúdos técnicos necessários ao uso de um sistema eletrônico de tramitação de documentos, que estava em fase de implantação para posterior uso obrigatório na organização.

Os três treinamentos estudados diferem entre si, em termos de conteúdos e desenhos instrucionais, porém os cursos voltados para os gerentes e líderes se assemelham em termos de público alvo e resultados organizacionais esperados. Os treinamentos gerenciais e de liderança visavam, além do impacto no comportamento dos egressos, efeitos nos processos de trabalho das equipes gerenciadas por esses egressos, foram ofertados, portanto, para um público alvo específico que lidera e influencia diretamente os resultados de suas respectivas equipes para que alcancem os resultados organizacionais esperados e valorizados pela organização (aumento de eficiência e eficácia). Enquanto, o SEI, é um treinamento de conteúdo técnico e administrativo, ofertado por um imperativo legal, que torna obrigatório o uso do sistema e que visava, no momento da pesquisa, em curto prazo, apenas apoiar a implantação dessa plataforma e mudar o comportamento dos egressos na comunicação e tramitação de documentos.

\section{Caracterização dos Participantes}

Todos os participantes da pesquisa eram egressos dos treinamentos estudados e que se dispuseram voluntariamente a responder o questionário. A amostragem para envio do instrumento foi não aleatória. Os dados utilizados nas análises de dados foram obtidos por meio da devolução dos questionários devidamente preenchidos pelos respondentes. O número total de participantes dos três treinamentos foi de 19.136 funcionários, dos quais $863(4,51 \%)$ eram egressos do curso Agente de Melhorias, 2.055 (10,74\%) do curso Gestão do Absenteísmo e 16.218 (84,75\%) do curso SEI. O disparo dos questionários foi feito pela própria organização e o sistema não conseguiu enviá-los para os 19.136 funcionários, por isso ele foi enviado para $10.592(55,35 \%)$ egressos desses cursos. A taxa final de retorno dos questionários respondidos por egressos dos três cursos foi de $28,09 \%$ (2.975). As amostras finais de participantes foram, portanto, não aleatórias, provenientes da devolução voluntária dos participantes.

Os participantes foram 2.975 (dois mil novecentos e setenta e cinco) funcionários da organização que realizaram pelo menos um dos três treinamentos e que responderam o questionário. Mais da metade dos respondentes $(59,2 \%)$ era do sexo masculino e $74,3 \%$ declararam ser casados ou estar em união estável. Em relação ao último nível de escolaridade concluído, predominaram participantes com ensino superior (45,1\%) ou especialização (41\%). Quanto ao tempo na função atual, 70,3\% relatou estar nessa função há no máximo seis anos; $43 \%$ dos participantes ocupam cargos funções de gestão/supervisão. Por fim, em relação à obrigatoriedade do treinamento, 50,8\% consideraram os treinamentos como de participação optativa e o restante como altamente recomendados.
As amostras de respondentes para cada treinamento foram as seguintes: $2.475(83,2 \%)$ para o curso Sistema Eletrônico de Informação, 425 (14,3\%) para o curso Gestão de Absenteísmo e o Incentivo à Presença Produtiva e 75 (2,5\%) para o curso Agente de Melhorias. A taxa de retorno voluntário dos questionários variou entre os cursos e foi maior para os egressos do treinamento SEI.

\section{Instrumentos de Coleta de Dados}

O instrumento de coleta de dados foi constituído por quatro blocos. O primeiro bloco, denominado "Valor do Treinamento", foi baseado na Escala de Valor Instrumental do Treinamento (EVIT) de Lacerda e Abbad (2003). O instrumento é composto de 14 itens associados a duas escalas, uma que avalia Valência (Importância) e outra Instrumentalidade (Utilidade). Dessa forma, os respondentes julgaram cada item por meio de duas escalas, sendo que uma delas avalia a importância de determinadas recompensas para a vida pessoal e profissional do respondente e a outra, a utilidade que ele atribui ao treinamento como meio para obter esses resultados (Abbad, Lacerda \& Pilati, 2012). A escala de Importância é composta por cinco pontos que variam de 1 (nada importante) a 5 (totalmente importante), enquanto que a escala de Utilidade varia de 1 (nada útil) a 5 (completamente útil). Por fim, foi acrescido ao final do bloco um item de Expectância proposto por Pilati (2004), cujo objetivo era avaliar a expectativa dos treinandos em atingir os valores por meio do treinamento; e dessa forma, calcular a força motivacional dos participantes. O item único é composto por uma escala tipo Likert de 0 (sem expectativa) a 10 (muita expectativa) ao qual o respondente avalia a sentença "O quanto você espera que as habilidades que você adquiriu no treinamento o ajudarão a alcançar os resultados descritos nos itens acima listados?". Dessa forma, Força Motivacional (FatMotiva) egressos poderia ser calculada a partir da multiplicação dos três componentes (valência, instrumentalidade e expectância).

O segundo bloco, denominado "Autoavaliação do Impacto do Treinamento no Trabalho" (Impacto), foi baseado na escala desenvolvida por Abbad (1999), composta de 12 itens avaliados em uma escala tipo Likert de cinco pontos, variando de 1 (Discordo totalmente da afirmativa) a 5 (Concordo totalmente com a afirmativa).

O terceiro bloco, denominado "Suporte ao Treinamento", foi baseado na Escala de Suporte à Transferência de Treinamento (EST) (Abbad, 1999). Utilizou-se a subdimensão Suporte Psicossocial (Suporte), composta por 16 itens ligados a fatores situacionais de apoio (nove itens) e consequências associadas ao uso de novas habilidades de trabalho (sete itens). Os itens são avaliados em uma escala tipo Likert de cinco pontos, variando de 1 (Nunca) a 5 (Sempre). Por fim, o quarto bloco corresponde aos dados pessoais e profissionais dos participantes: sexo, estado civil, último nível de escolaridade, área da última formação acadêmica, ano que concluiu a formação, tempo em que trabalhava na organização e na função atual, se ocupa cargo de gestão ou supervisão e se o treinamento foi optativo (voluntário) ou obrigatório (altamente recomendado pelo gestor).

\section{Procedimentos de Coleta de Dados}

Para realizar a coleta de dados no formato online, o questionário, autoaplicável, foi digitalizado para o Google Forms e repassado para uma lista de egressos dos três treinamentos em um e-mail com uma carta de sensibilização. Após 15 dias, outro e-mail foi enviado à lista, com mensagem de agradecimento aos que responderam e reiterando o pedido aos demais para que participassem da pesquisa preenchendo o referido questionário. Os cuidados éticos 
necessários foram tomados, garantindo aos participantes o caráter sigiloso das respostas individuais, bem como a participação voluntária na pesquisa e a devolução dos resultados agrupados.

O questionário foi enviado para 10.592 egressos dos treinamentos por meio de e-mail institucional e a taxa final de retorno fo de $28,09 \%$ (2.975). Os egressos preencheram o questionário após realização do treinamento, porém o tempo entre o treinamento e a participação na pesquisa foi variável. Os dados coletados foram salvos automaticamente pelo Google Forms, em planilha compatível com Excel, e posteriormente exportados para o SPSS versão 21.

\section{Cuidados éticos}

Foram garantidos aos respondentes o sigilo das respostas individuais e a participação voluntária na pesquisa, mediante consentimento livre e esclarecido. Os pesquisadores se responsabilizaram pelo tratamento agrupado dos resultados e cuidados éticos, de tal modo que o respondente, ao preencher o questionário e enviar as suas respostas, consentiam o uso dos dados para as finalidades da pesquisa. Essas informações estão contidas no texto original nas páginas destinadas aos procedimentos de coleta de dados e em mensagem enviada pela área responsável pela educação corporativa aos participantes da pesquisa.

\section{Procedimento de Análise de Dados}

A análise de dados foi realizada em quatro etapas. Na primeira, foi feita a verificação dos pressupostos estatísticos (Tabachnick \& Fidell, 2001; Hair, Black, Babin, Anderson, \& Tatham, 2010). Foram realizadas análises estatísticas preliminares e de caráter exploratório por meio de testes de comparação entre médias (testes t e análises de variância), análises fatoriais exploratórias e análises de regressão múltipla. Foram separados os dados para toda a amostra ( $N=2825)$, a amostra do curso do SEI ( $N=2338)$ e a amostra do curso de gestão ( $N=487$ ). Como o número de respondentes do Curso SEl era superior ao curso de gestão, foi selecionada uma amostra aleatória de 500 respondentes do curso SEI para avaliação do efeito moderador do curso. A segunda etapa da análise de dados consistiu em verificar o ajuste do modelo de investigação e foi feita em cada um dos três bancos após todo o procedimento de limpeza de dados. Foram feitas avaliações dos modelos de medida e do modelo estrutural (Tabachnick \& Fidell, 2001; Hair, Black, Babin, Anderson, \& Tatham, 2010).

Os modelos de equação estrutural, recomendados para testar estruturas fatoriais, por meio de análises fatoriais confirmatórias e para analisar relacionamentos entre múltiplas variáveis latentes e observadas ao mesmo tempo, tal como almejado por este estudo, foram estimados no programa AMOS 20 por máxima verossimilhança, para verificação do ajuste dos modelos a partir dos indicadores apontados por Byrne (2010) e Kline (2010): GFI (Goodness-of-Fit Index), CFI (Comparative Fit Index) e RMSEA. O modelo proposto foi comparado a modelos alternativos, pois tal prática constitui teste mais rigoroso do que a análise isolada do ajuste (Hair, Black, Babin, Anderson \& Tatham, 2010).

A terceira etapa consistiu na verificação dos testes de hipóteses. O modelo de mediação deste estudo é considerado complexo por ter mais de uma variável como mediadora (Preacher, Zyphur, \& Zhang, 2010). Assim, a verificação de mediação nesse estudo foi feita por meio de equações estruturais, e foram verificadas a magnitude e significância dos efeitos indiretos por meio de bootstrapping (método de reamostragem que utiliza os mesmos dados para estimar distribuições amostrais de parâmetros). Os efeitos indiretos são estimados a partir de uma amostra da população e são produtos das estimativas dos coeficientes de regressão. Efeitos indiretos significativos são uma medida análoga à utilização de outros métodos que indicam a significâncias dos efeitos de interação da regressão (Preacher \& Selig, 2012). Para isso, foram realizadas várias análises com submodelos com o objetivo de verificar o sinal, magnitude e significância dos coeficientes, além de verificar a relação de mediação (Preacher, Zyphur, \& Zhang, 2010; Preacher \& Selig, 2012).

A quarta etapa teve por objetivo verificar o efeito moderador do curso. Para isso foi criada uma variável dicotômica denominada Curso, na qual foram agrupados em uma única categoria os dois treinamentos voltados à gestão (Agente de Melhorias e Gestão do Absenteísmo e o Incentivo à Presença Produtiva) e em outra o curso para uso do SEI e foi feita a comparação do ajuste do modelo de investigação em amostras do SEI e dos dois cursos de gestão, utilizando a estatística qui-quadrado. Também foram realizados testes $t$ de student para fornecer tamanhos de efeitos para a extensão da moderação ( $d$ de Cohen).

\section{Resultados}

\section{Evidências de Validade da Escala}

As escalas, submetidas a análises fatoriais e análise de consistência interna (Alpha de Cronbach), mostraram evidência de validade e fidedignidade, conforme Tabela 1.

Tabela 1

Evidências psicométricas de validade das escalas utilizadas

\begin{tabular}{|c|c|c|c|c|c|}
\hline \multirow{2}{*}{ Instrumento } & \multirow{2}{*}{$\begin{array}{l}\text { Número } \\
\text { de itens }\end{array}$} & \multirow{2}{*}{ Alfa } & \multirow{2}{*}{$\begin{array}{l}\text { \% de Variância } \\
\text { total explicada }\end{array}$} & \multicolumn{2}{|c|}{ Cargas fatoriais } \\
\hline & & & & Máximo & Mínimo \\
\hline $\begin{array}{l}\text { Instrumentalidade } \\
\text { do Treinamento }\end{array}$ & 14 & 0,945 & 55,463 & 0,830 & 0,649 \\
\hline $\begin{array}{l}\text { Valência do } \\
\text { Treinamento }\end{array}$ & 14 & 0,944 & 54,791 & 0,817 & 0,624 \\
\hline $\begin{array}{l}\text { Suporte Psicos- } \\
\text { social à Transfe- } \\
\text { rência }\end{array}$ & 9 & 0,910 & 54,212 & 0,873 & 0,549 \\
\hline $\begin{array}{l}\text { Impacto do } \\
\text { Treinamento no } \\
\text { Trabalho em am- } \\
\text { plitude }\end{array}$ & 12 & 0,935 & 54,995 & 0,830 & 0,547 \\
\hline
\end{tabular}

Além desses instrumentos, para a mensuração da Expectância, foi utilizado o item único como referido anteriormente. Da escala original de Suporte Psicossocial à Transferência com 16 itens, restaram nove itens, após as análises da estrutura empírica da escala, pois sete itens tinham correlações baixas e estatisticamente não significativas com as demais variáveis.

\section{Modelo Estrutural e Teste de Hipóteses}

A verificação do ajuste do modelo foi realizada de forma separada para os grupos (amostra total, curso SEl e curso Gestão). O modelo testado se encontra na Figura 3. Os índices de ajuste do modelo estrutural proposto são considerados razoáveis e são apresentados na Tabela 2. Os modelos reespecificados apresentam duas correlações entre os erros dos itens 8 e 9 e 10 e 11 do instrumento de suporte, indicados pelo software AMOS. Tais erros indicam provavelmente problemas vinculados a tais itens. Os modelos testados apresentam bons índices de ajuste (Kline, 2010), o que indica que as relações apresentadas possuem respaldo empírico. Após a testagem geral foram verificadas as hipóteses estipuladas no modelo. 
Tabela 2

Índices de Adequação do Modelo

\begin{tabular}{|c|c|c|c|c|c|c|c|c|c|c|}
\hline Modelo/índices & $\chi^{\prime \prime}$ & g.l. & $\chi^{\prime \prime}$ p. $\lambda$ & $\mathrm{NFI}$ & TLI & CFI & GFI & $\begin{array}{l}\text { RMSEA } \\
\text { (IC 95\%) }\end{array}$ & $\Delta \chi^{2 *}$ & $\Delta g . I^{*}$ \\
\hline Modelo amostra geral & 5406,41 & 206 & 26,2 & 0,91 & 0,90 & 0,91 & 0,89 & $\begin{array}{c}0,09 \\
(0,08-0,09)\end{array}$ & & \\
\hline Modelo SEI & 4083,16 & 204 & 20,01 & 0,91 & 0,90 & 0,91 & 0,89 & $\begin{array}{c}0,09 \\
(0,08-0,09)\end{array}$ & & \\
\hline Modelo SEI reespecificado & 1132,62 & 205 & 5,53 & 0,88 & 0,89 & 0,91 & 0,91 & $\begin{array}{c}0,09 \\
(0,09-0,10)\end{array}$ & 525,42 & 73 \\
\hline Modelo Gestão reespecificado & 607,2 & 132 & 4,60 & 0,93 & 0,92 & 0,93 & 0,91 & $\begin{array}{c}0,08 \\
(0,07-0,09)\end{array}$ & 525,42 & 73 \\
\hline
\end{tabular}

Nota. ${ }^{*} p<0,001 ;$ g.l. ,= graus de liberdade; IC = intervalo de confiança (95\%); $\Delta \chi^{2}=$ diferença de qui-quadrado entre modelo SEl e modelo Gestão.; $\Delta g . I .=$ diferença de graus de liberdade entre modelo SEI e modelo Gestão.

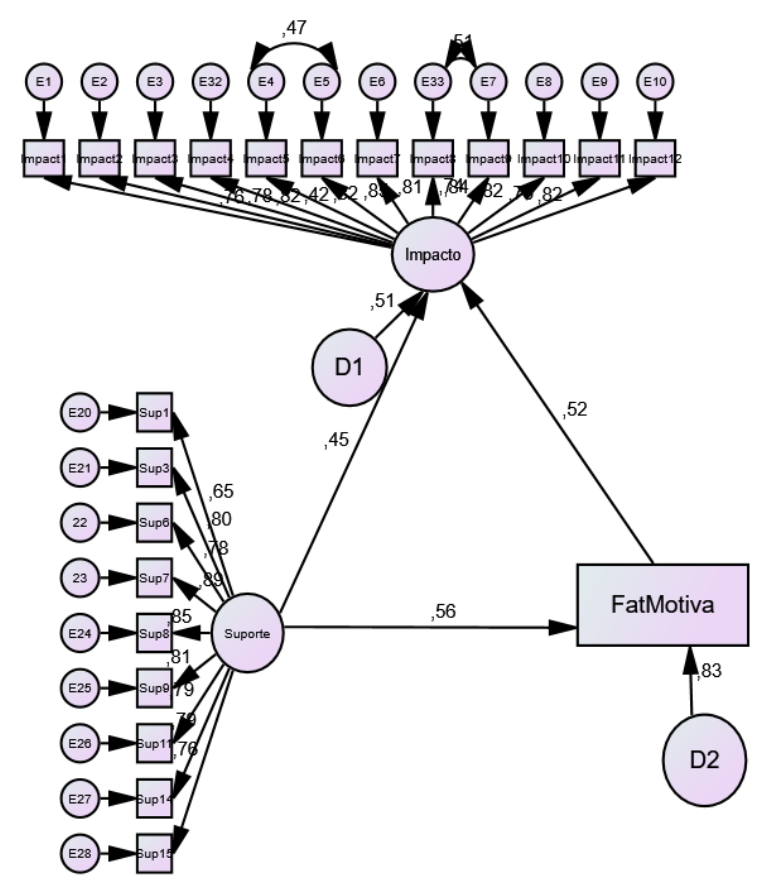

Figura 3. Modelo testado nas três amostras

A primeira hipótese foi totalmente corroborada. O efeito da relação entre suporte a transferência e força motivacional é alto, positivo e significativo T1 ( $\beta=0,56, p<0,001)$. Em todos os modelos testados, a magnitude do efeito apresentou valores semelhantes. $O$ efeito da relação entre força motivacional e impacto do treinamento também é positivo e significativo $(\beta=0,41 ; p<0,001)$. $O$ efeito da relação entre suporte à transferência e impacto do treinamento também é alto e positivo $(\beta=0,55, p<0,001)$. Portanto, a segunda e terceira hipóteses foram corroboradas. Os dados sobre a magnitude do efeito nas relações testadas nos três modelos se encontram na Tabela 3.

Tabela 3

Magnitude do efeito das relações testadas

\begin{tabular}{|c|c|c|c|c|c|c|}
\hline \multirow{2}{*}{$\begin{array}{l}\text { Relação } \\
\text { Índices/modelos }\end{array}$} & \multicolumn{2}{|c|}{$\begin{array}{l}\text { Suporte/ } \\
\text { Impacto }\end{array}$} & \multicolumn{2}{|c|}{$\begin{array}{l}\text { Suporte/ } \\
\text { Força motiva- } \\
\text { cional }\end{array}$} & \multicolumn{2}{|c|}{$\begin{array}{l}\text { Força mo- } \\
\text { tivacional/ } \\
\text { Impacto }\end{array}$} \\
\hline & $\beta$ & $p$ & $\beta$ & $p$ & $\beta$ & $p$ \\
\hline Modelo amostra geral & 0,51 & 0.001 & 0,61 & 0.001 & 0,48 & 0.001 \\
\hline Modelo SEI & 0,52 & 0,001 & 0,56 & 0,000 & 0,47 & 0,000 \\
\hline Modelo SEI reespecificado & 0,45 & 0.001 & 0,56 & 0.001 & 0,52 & 0.001 \\
\hline Modelo Gestão & 0,34 & 0,000 & 0,56 & 0,001 & 0,59 & 0,000 \\
\hline $\begin{array}{l}\text { Modelo Gestão reespecifi- } \\
\text { cado }\end{array}$ & 0,33 & 0.001 & 0,56 & 0.001 & 0,60 & 0.001 \\
\hline
\end{tabular}

A quarta hipótese, se relacionava ao teste de mediação. A literatura afirma que quando a variável mediadora é incluída na equação de regressão, há redução ou é neutralizado o impacto da variável independente sobre a dependente (Tabachnick \& Fidell, 2001). Os resultados das análises de mediação são apresentados na Tabela 4.

Tabela 4

Resultado do teste de mediação para a variável dependente Impacto de Treinamento no Trabalho*

\begin{tabular}{|c|c|c|c|c|c|c|}
\hline \multirow[b]{2}{*}{ Variáveis } & \multicolumn{2}{|c|}{ Amostra total } & \multicolumn{2}{|c|}{ Amostra SEI } & \multicolumn{2}{|c|}{ Amostra Gestão } \\
\hline & $\beta$ & $\begin{array}{l}\text { Efeito } \\
\text { indireto }\end{array}$ & $\beta$ & $\begin{array}{l}\text { Efeito } \\
\text { indireto }\end{array}$ & $\beta$ & $\begin{array}{l}\text { Efeito } \\
\text { indireto }\end{array}$ \\
\hline Suporte & $0,51 * *$ & & $0,45^{* *}$ & & $0,33 * *$ & \\
\hline $\begin{array}{l}\text { Força } \\
\text { motivacional }\end{array}$ & $0,48^{* *}$ & & $0,52 * *$ & & $0,60 * *$ & \\
\hline $\begin{array}{l}\text { Suporte/Força } \\
\text { motivacional }\end{array}$ & $0,61^{* *}$ & $0,57 * *$ & $0,56^{* *}$ & $0,52 * *$ & $0,56 * *$ & $0,52 * *$ \\
\hline
\end{tabular}

Os resultados do teste de mediação da força motivacional na relação entre suporte a transferência e impacto do treinamento no trabalho foram significativos e presentes em todos os modelos testados. Os resultados demonstram indícios de mediação (Baron \& Kenny, 1986) e os efeitos indiretos foram corroborados pelo uso do bootstrapping, dando suporte à hipótese de mediação. Os indicadores das relações entre suporte à transferência e impacto do treinamento demonstraram mediação completa pela variável força motivacional, ou seja, a relação entre a variável preditora e a variável critério se tornou insignificante após a inclusão da variável mediadora (Hair et al, 2010).

A quinta hipótese diz respeito ao efeito moderador do curso nas relações entre suporte a transferência, força motivacional e impacto do treinamento. Para isso, foi feita a comparação do ajuste do modelo de investigação nas amostras do SEl e dos cursos de gestão, utilizando a estatística qui-quadrado. Uma mudança não significativa no valor do qui-quadrado, ou $\Delta \chi^{2}(\Delta d f)$, indica que o modelo não varia conforme o tipo de curso, ou seja, não há efeito moderador do tipo de curso sobre as relações entre as variáveis do modelo. Caso ocorra uma mudança significativa nesse valor fica evidenciado o efeito moderador do curso. Também foram realizados testes $t$ de student para fornecer tamanhos de efeitos para a extensão da moderação ( $d$ de Cohen). De acordo com os dados da Tabela 2, houve uma diferença significativa no Qui-quadrado de 525,42 com a diferença também significativa de 73 graus de liberdade. Portando, há efeito moderador do curso no ajuste do modelo testado. 0 curso modera as relações testadas entre suporte a transferência, força motivacional e impacto do treinamento. 


\section{Discussão}

O objetivo deste artigo foi avaliar a influência de percepções de Suporte Psicossocial à Transferência (SUP) e Força Motivacional (FatMotiva) sobre Impacto do Treinamento no Trabalho (IMTT), a partir da avaliação de três treinamentos corporativos (Agente de Melhorias, Gestão do Absenteísmo e Incentivo à Presença Produtiva e SEI, Sistema Eletrônico de Informação). Esse objetivo foi atingido, uma vez que se verificou a predição de FatMotiva e SUP sobre IMTT, bem como a mediação de SUP na relação entre FatMotiva e IMTT.

Observou-se que Suporte Psicossocial é preditor de Impacto do treinamento no trabalho, resultado similar ao encontrado em estudos que adotaram as mesmas medidas Abbad (1999); Meneses e Abbad (2003) ou medidas correlatas de Al-Eisa et al. (2009); Bhatti, Ali, Isa e Battour (2014); Schindler e Burkholder (2016) e W. Silva (2008). Depieri (2006) também encontrou que SUP é um preditor de Impacto do Treinamento no Trabalho, porém, ao contrário dos resultados aqui encontrados, as características individuais, tais como motivação, não se mostraram preditoras de IMTT.

Os resultados da presente pesquisa vão ao encontro dos achados de C. R. E. Silva (2007) uma vez as duas dimensões de FatMotiva (valência e instrumentalidade) foram também preditoras de impacto. Motivação também é tida como preditora de IMTT, assim como nos estudos de Lacerda e Abbad (2003) e C. R. E. Silva (2007), Hutchins, Nimon, Bates e Holton (2013), e Schindler e Burkholder (2014) que utilizaram medidas correlatas de IMTT (Transferência, intenção de transferir). Grohmann, Beller e Kauffeld (2014) encontraram motivação para transferir como variável mediadora total da relação entre características do treinamento e transferência de treinamento. Al-Eisa et al. (2009) encontraram que, quando a motivação para aprender foi inserida no modelo de regressão múltipla, suporte do supervisor ainda tinha um efeito estatisticamente significativo sobre a intenção de transferir, mas a motivação para aprender mediou parcialmente a relação entre suporte do supervisor e intenção de transferir. Além disso, encontraram uma mediação completa de suporte do supervisor sobre a relação entre autoeficácia e intenção de transferir. A presente pesquisa avança a discussão sobre a relação de motivação como mediadora de suporte para transferência e impacto do treinamento no trabalho, ao encontrar resultados significativos e que atendem ao chamado de Gegenfurtner et al. (2014) que sugerem mais pesquisas sobre o assunto.

Foi observada relação positiva entre FatMotiva e SUP, bem como dessas duas variáveis na predição de IMTT, evidenciando, também, a importância da relação entre Suporte e as medidas correlatas de Impacto do Treinamento no trabalho. Corroborando com Bell et al. (2017), que concluíram após revisão que suporte dos pares e do supervisor podem determinar até que ponto as competências recém-adquiridas serão aplicadas no trabalho, assim como o fato do contexto de trabalho (suporte, por exemplo) influenciar nas atividades de desenvolvimento e em sua efetividade, uma vez que pode aumentar ou diminuir a motivação dos participantes para aprender e aplicar o que aprenderam no treinamento. Esses autores também apontaram para a influência que fatores do contexto, como suporte e experiências pré-treinamento exercem sobre a condição do funcionário participar ou não de um treinamento não-obrigatório.

As principais contribuições teóricas deste estudo consistem no reforço do efeito preditor das variáveis de Suporte Psicossocial à Transferência e de Força Motivacional sobre o Impacto do Treinamento no Trabalho, bem como a relação de mediação que a variável Força Motivacional estabelece entre Suporte Psicossocial e
Impacto do Treinamento no Trabalho. As contribuições metodológicas englobam as evidências de validade das três escalas utilizadas nesse estudo, confirmando resultados anteriores; a análise de mediação tendo como variável mediadora Força Motivacional e o uso de equações estruturais para teste do modelo.

O efeito moderador do tipo de treinamento sobre as relações entre as variáveis suporte, força motivacional e a variável critério impacto do treinamento no trabalho parecem indicar a importância dos resultados esperados pela organização e do público-alvo do treinamento na predição da eficácia de cursos em ambientes corporativos. $\mathrm{O}$ efeito moderador do tipo de treinamento, provavelmente não se deveu, nesta pesquisa, à qualidade instrucional dos cursos avaliados, tampouco à modalidade de entrega (presencial ou a distância), mas ao nível de resultados esperados pela organização como efeito desses treinamentos e ao poder ou autoridade dos participantes para produzir esses resultados. As hipóteses iniciais deste estudo que pautaram a escolha dos treinamentos a serem estudados baseavam-se na suposição de que os cursos gerenciais seriam mais motivadores, obteriam maior suporte e produziriam maior impacto do que SEI, um treinamento específico, voltado a habilidades administrativas, ofertado para um público alvo bastante amplo.

$\mathrm{Na}$ organização estudada, enquanto os treinamentos gerenciais e de liderança visavam, além do impacto no comportamento dos egressos, efeitos nos processos de trabalho das equipes gerenciadas por esses egressos, o treinamento SEl visava apenas a implantação obrigatória do sistema pela organização. As lideranças, ao possuírem autoridade e capacidade de influenciar diretamente os resultados de suas respectivas equipes, teriam maior motivação e receberiam maior suporte do que os participantes do curso de conteúdo técnico e administrativo, ofertado pela organização, em função de legislação que estabeleceu a obrigatoriedade do uso do SEI e prazos para a sua implantação. Essas hipóteses foram confirmadas e os resultados contribuem para ampliar a discussão sobre as variáveis moderadoras de interesse em TD\&E, ao inserir resultados esperados e tipo de público alvo como aspectos relevantes a serem investigados em pesquisas futuras. Organizações precisam, de acordo com esses achados, preocupar-se em conferir valor, instrumentalidade e expectativa de sucesso aos participantes de treinamentos, adotando análises de necessidades de treinamento que levem em conta aspectos motivacionais e o papel do treinamento no alcance de resultados almejados pelos indivíduos e equipes de trabalho.

Por fim, nas contribuições práticas tem-se a importância do suporte psicossocial à transferência, com atenção de gestores para disseminação de novas aprendizagens, bem como a necessidade de desenvolver uma cultura de aprendizagem na organização em que os pares estimulem a aquisição de novos conhecimentos e assim, egressos de treinamentos possam transmitir os conhecimentos adquiridos. É preciso evidenciar os benefícios do treinamento para os egressos e organização, estimulando o aumento da motivação para aprender e transferir.

O desenvolvimento de uma cultura de aprendizagem contínua e de educação continuada e ao longo de toda a vida funcional é de grande importância para a educação corporativa, pois aumentará as chances de produzir melhorias no desempenho de egressos de treinamento e impactos positivos sobre processos e resultados organizacionais valorizados pela organização. Além disso, na análise de necessidades de treinamento, é preciso identificar as condições materiais necessárias à transferência de treinamentos para o trabaIho, de modo a diminuir as chances de que as novas aprendizagens 
sejam esquecidas pela falta de oportunidade de praticá-las no local de trabalho.

Dentre as limitações do estudo, cabe mencionar inicialmente que a pesquisa foi realizada em uma única organização, reduzindo a possibilidade de generalização dos resultados para outros contextos. Além disso, houve a impossibilidade de aplicação do questionário heteroavaliativo de Impacto do Treinamento no Trabalho, que poderia gerar resultados comparativos com os de autoavaliação. Ademais, as avaliações foram feitas após o treinamento, não podendo comparar a Força Motivacional dos participantes antes deste iniciar. Por fim, os construtos de IMTT, FatMotiva e SUP possuem diversas medidas correlatas, porém não se sabe se possuem validade convergente ou divergente.

De acordo com os resultados apresentados e discutidos, propõe-se como agenda de pesquisa para estudos futuros: (1) Aplicar os instrumentos utilizados neste estudo em outras organizações, treinamentos e tipos de cursos (presencial e a distância), a fim de confirmar sua confiabilidade e replicação; (2) Aplicar questionários de auto e heteroavaliação nos participantes e em seus gestores; (3) Utilizar medidas de Impacto do Treinamento no Trabalho em Profundidade, baseadas nos objetivos de treinamento; (4) Incluir avaliações antes, durante e depois do treinamento, afim de avaliar as variações da Força Motivacional e as percepções em relação ao Suporte à Transferência de Treinamento; (5) Acrescentar no modelo de avaliação outras características individuais (autoeficácia, valores, etc) como preditoras do Impacto do Treinamento no Trabalho; (6) Analisar a relação de mediação de força motivacional e impacto do treinamento no trabalho em contextos diferentes; e (7) Construir e buscar evidência de validade de medida de expectância.

\section{Referências}

Abbad, G. S. (1999). Um modelo integrado de avaliação do impacto do treinamento no trabalho - IMPACT. Tese de Doutorado, Universidade de Brasília, Brasília, DF, Brasil. Recuperado de http://repositorio.unb.br/bitstream/10482/17469/1/2014_DanieleMartinsVitoria.pdf

Abbad, G.S., Borges-Andrade, J.E., \& Sallorenzo, L. E. (2004). Self-assessment of training impact at work: validation of a measurement scale. Interamerican Journal of Psychology, 38(2), 277-288. Recuperado de https://www.researchgate.net/publication/28317079_Self-Assessment_of_Training_Impact_ at_Work_Validation_of_a_Measurement_Scale

Abbad, G. S., Lacerda, E. R. M., \& Pilati, R. (2012). Medidas de motivação e valor instrumental do treinamento. In G. S. Abbad, L. Mourão, P. P. M. Meneses, T. Zerbini, J. E. Borges-Andrade, \& R. Vilas-Boas (eds) Medidas de Avaliação em Treinamento, Desenvolvimento e Educação: ferramentas para gestão de pessoas. (pp. 198-215). Porto Alegre: Artmed.

Al-Eisa, A. S., Furayyan, M. A., \& Alhemoud, A. M. (2009). An empirical examination of the effects of self-efficacy, supervisor support and motivation to learn on transfer intention. Management decision, 47(8), 1221-1244. doi: 10.1108/00251740910984514

Baldwin, T. T., \& Ford, J. K. (1988). Transfer of training: a review and directions for future research. Personnel Psychology, 41(1), 63-105. doi: 10.1111/j.17446570.1988.tb00632.x

Baron, R. M., \& Kenny, D. A. (1986). The Moderator-Mediator Variable Distinction in Social Psychological Research: conceptual, strategic, and statistical considerations. Journal of Personality and Social Psychology, 51(6), 1173-1182. doi: 10.1037/0022-3514.51.6.1173

Bhatti, M. A., Ali, S., Isa, M., Faizal, M., \& Battour, M. M. (2014). Training transfer and transfer motivation: the influence of individual, environmental, situational, training design, and affective reaction factors. Performance Improvement Quarterly, 27(1), 51-82. doi: 10.1002/piq.21165

Bräten, I., \& Ferguson, L. E. (2015). Beliefs about sources of knowledge predict motivation for learning in teacher education. Teaching and Teacher Education, 50, 13-23. doi: 10.1016/j.tate.2015.04.003

Bell, B. S., Tannenbaum, S. I., Ford, J. K., Noe, R. A., \& Kraiger K. (2017). 100 Years of Training and Development Research: What We Know and Where We Should Go. Journal of Applied Psychology, 1-19. doi: 10.1037/apl0000142
Burke, L. A., \& Hutchins, H. M. (2007). Training transfer: an integrative literature review. Human Resource Development Review, 6(3), 263-296. doi: 10.1177/1534484307303035

Chiaburu, D. D., Van Dam, K, \& Hutchins, H. M. (2010). Social support in the workplace and training transfer: a longitudinal analysis. International Journal of Selection and Assessment, 18(2), 187-200. doi: 10.1111/j.1468-2389.2010.00500.x

Colquitt, J. A., LePine, J. A., \& Noe, R. N. (2000). Toward and integrative theory of training motivation: a meta-analytic path analysis of 20 years of research. Journal of Applied Psychology, 85(5), 678-707. doi: 10.1037//0021-9010. g5.5.678

Curado, C., Henriques, P. L., \& Ribeiro, S. (2015). Voluntary or mandatory enrollment in training and the motivation to transfer training. International Journal of Training and Development, 19(2), 98-109. doi: 10.1111/ijtd.12050

Depieri, M. A. (2006). Impacto de educação corporativa: Educação continuada em processos educativos - FORFOR. Dissertação de Mestrado, Universidade de Brasília, Brasília, DF, Brasil. Recuperado de: http://bdtd.ibict.br/vufind/ Record/UNB_4f416f315a942d1355a769437a45ea9c

Gegenfurtner, A., Veermans, K., Festner, D., \& Gruber, H. (2009). Motivation to transfer training: an integrative literature review. Human Resource Development Review, 8(3), 403-423. doi: 10.1177/1534484309335970

Gegenfurtner, A. (2011). Motivation and transfer in professional training: a meta-analysis of the moderating effects of knowledge type, instruction and assessment conditions. Educational Research Review, 6(3), 153-168. doi: 10.1016/j. edurev.2011.04.001

Grohmann, A., Beller, J., \& Kauffeld, S. (2014). Exploring the critical role of motivation to transfer in the training transfer process. International Journal of Training and Development, 18(2), 84-103. doi: 10.10.1111/ijtd.12030

Hutchins, H. M, Nimon, K., Bates, R., \& Holton, E. (2013). Can the LTSI predict transfer performance? Testing intent to transfer as a proximal transfer of training outcome. International Journal of Selection and Assessment, 21(3), 251-263. doi: 10.1111/ijsa.12035

Kochoian, N., Raemdonck, I., Frenay, M., \& Zacher, H. (2016). The role of age and occupational future time perspective in workers' motivation to learn. Vocations and Learning, 1-19. doi: 10.1007/s12186-016-9160-9

Kodwani, A. D. (2017). Decoding training effectiveness: the role of organizational factors. Journal of Workplace Learning, 29(3), 200-216. doi: 10.1108/ JWL-05-2016-0038

Krishnamani, S., \& Haider, Y. (2016). Transfer of learning from executive education programs in developing economies: the key role of motivation. Development and Learning in Organizations: An International Journal, 30(1), 15-18. doi: 10.1108/DLO-05-2015-0051

Lacerda, E. R. M., \& Abbad, G. (2003). Impacto do treinamento no trabalho: investigando variáveis motivacionais e organizacionais como preditoras. Revista de Administração Contemporânea, 7(4), 77-96. doi: 10.1590/ S1415-65552003000400005

Lacerenza, C. N., Reyes, D. L., Marlow, S. L., Joseph, D. L., \& Salas, E. (2017). Leadership Training Design, Delivery, and Implementation: A Meta-Analysis. Journal of Applied Psychology, 102(12), 1686-1718. doi: 10.1037/apl0000241

López, M. G. M., \& Aguilar, A. P. (2013). Emotions as learning enhancers of foreign language learning motivation. Profile Issues in Teachers Professional Development, 15(1), 109-124. Recuperado de https://revistas.unal.edu.co/ index.php/profile/article/view/37872/40579

McEown, M. S., Noels, K. A., \& Saumure, K. D. (2014). Students' self-determined and integrative orientations and teachers' motivational support in a Japanese as a foreign language context. System, 45, 227-241. doi: 10.1016/j. system.2014.06.001

Meneses, P. P. M., \& Abbad, G. S. (2003). Preditores individuais e situacionais de auto e heteroavaliação de impacto de treinamento no trabalho. Revista de Administração Contemporânea, 7 (Edição Especial), 185-204. doi: 10.1590/ S1415-65552003000500010

Noe, R. A. (1986). Trainees' attributes and attitudes: neglected influences on training effectiveness. Academy of Management Review, 11(4), 736-749. doi: $10.2307 / 258393$

Pilati, R. (2004). Modelo de efetividade do treinamento no trabalho: aspectos dos treinandos e moderação do tipo de treinamento. Tese de Doutorado, Universidade de Brasília, Brasília, DF, Brasil.

Reinhold, S., Gegenfurtner, A., \& Lewalter, D. (2018). Social support and motivation to transfer as predictors of training transfer: testing full and partial mediation using meta-analytic structural equation modelling. International Journal of Training and Development. 1-14. ISSN 1360-3736. doi: 10.1111/ ijtd.12115 
Santos, A. A. A., \& Mognon, J. F. (2016). Motivation Assessment Scale for Learning in Higher Education (EMAPRE-U): Validity Evidence. Psico-USF, 21(1), 101-110. doi: 10.1590/1413-82712016210109

Silva, C. R. E. (2007). Orientação profissional: utilidade, valor e impacto na gestão da carreira e na vida pessoal. Dissertação de Mestrado, Universidade de Brasília, Brasília, DF, Brasil. Recuperado de http://repositorio.unb.br/bitstream/10482/2810/1/2007_CarlosRobertoErnestodaSilva.pdf

Silva, W. (2008). O impacto das ações de capacitação na atuação gerencial: um estudo de caso no setor público. Tese de Doutorado. Universidade de Brasília, Brasília. Recuperado de http://repositorio.unb.br/handle/10482/3980

Wingo, M. T., Thomas, K. G., Thompson, W. G., \& Cook, D. A. (2015). Enhancing motivation with the "virtual" supervisory role: a randomized trial. BMC Medical Education, 15, 1-7. doi: 10.1186/s12909-015-0348-8

Zerbini, T., \& Abbad, G. (2011). Transferência de treinamento e impacto do treinamento no trabalho: análise crítica da literatura. Revista Psicologia Organizações e Trabalho, 10(2), 97-111. Recuperado de https://periodicos. ufsc.br/index.php/rpot/article/view/22212 